\title{
Summary of research on pretreatment methods of recycled aggregate
}

\author{
Song Yufeng, ${ }^{1, *}$, Zhang Enqiang ${ }^{1}$ \\ ${ }^{1}$ China Railway 12th Bureau Group Co., LTD, 030024, China, Shanxi
}

\begin{abstract}
The recycled aggregate obtained by simple crushing and screening of waste concrete has a rough surface and many protruding edges and corners, and most of the aggregate surface is attached with old mortar, when directly used as aggregate in concrete, the water demand is large, the concrete strength and elastic modulus are low, and the durability is poor. Therefore, proper treatment of recycled aggregate is required.This article summarizes the research status of the pretreatment methods of mechanical strengthening, heating grinding, solution impregnation, cement slurry impregnation, carbonization treatment, nano-modification, microbial modification and water cleaning etc., and simply analyzes the advantages and disadvantages of various treatment methods, which provide a reference for the research of recycled aggregate processing methods.
\end{abstract}

\section{Introduction}

With the continuous development of the construction industry and the frequent occurrence of natural disasters such as earthquakes, more and more construction waste has accumulated, occupying a large amount of land resources, and also causing certain pollution to the environment. At the same time, China is a country with a large consumption of sand and gravel. In the past few years, the amount of sand and gravel has exceeded 15 billion tons per year. In 2020, the amount of sand and gravel has reached 17.826 billion tons. Natural sand and gravel that can be mined are getting less and less. The sand and gravel resources in some areas are very scarce, and the price of sand and gravel is constantly rising, which directly affects the progress and cost of project construction. Therefore, recovering sand and gravel aggregates from construction waste is the current focus of the construction industry.

A large amount of waste concrete will be produced after building disintegration, the recycling aggregate (RA) refers to the concrete aggregate obtained after the waste concrete is broken, graded, cleaned and adjusted according to the requirements of coarse aggregate particle grading according to the national standard[1].

The recycled aggregate particles prepared by simple crushing and screening processes have rough surface and prominent edges and corners[2], meanwhile, the surface of the aggregate is wrapped with much old hardened cement mortar, which is the main difference between the recycled aggregate and natural aggregate[3]. The quantity and quality of the attached hardened old cement mortar are the main factors affecting the physical properties of recycled aggregates[4], the old cement mortar has a porous micro-structure and many micro-cracks, which can adversely affect the strength of the interfacial transition zone when used to produce concrete[5]. At the same time, in the crushing process of waste concrete, a large amount of mechanical damage will be accumulated. Compared with natural aggregate, recycled aggregate has high water absorption rate, large porosity, low apparent density and high crushing value[6,7]. When directly used to produce concrete, more water is required, the hardened concrete has low strength and elastic modulus, meanwhile, the durability indexes such as permeability resistance, frost resistance and chloride ion permeability resistance are lower than ordinary concrete[8]. Therefore, the recycled aggregate needs to be pretreated before it is used.

\section{Pretreatment method of recycled aggregate}

Through appropriate pretreatment methods, the performance of RA can be improved and enhanced. The common strengthening and modification treatment methods include physical strengthening 、 chemical strengthening 、 carbonization treatment nano-material modification microbial deposition and water cleaning methods.

\subsection{Physical strengthening method}

Physical reinforcement refers to the further treatment of the RA obtained by physical means. The improvement of RA performance is mainly reflected in two aspects: one is to improve the aggregate grain shape through impact and friction, it can reduce the crushing value of aggregate, reduce the stress concentration inside

\footnotetext{
*Song Yufeng: 1246699749@qq.com
} 
concrete, and reduce the probability of crack. The second is to further reduce the old cement mortar wrapped on the aggregate surface, reduce porosity and water absorption of RA, and improve the roughness of aggregate surface. There are many specific methods of physical strengthening, there are roughly two ways, one is the mechanical strengthening method through the collision and friction between particles and machinery or between particles. The other is the heating grinding method which decomposes cement mortar and grinds it by heating. There are many specific methods of physical strengthening, which can be roughly divided into two methods. One is the mechanical strengthening method through collision and friction between particles and machinery or between particles, and the other is to decompose cement mortar and grind it by heating.

\subsubsection{Mechanical strengthening}

The mechanical strengthening method generally uses ball mills, rod mills and other mechanical equipment to process RA. It uses the collision and friction which between particles or between mechanical equipment and particles to remove the old mortar attached from the surface and grind off the protruding edges and corners, thereby improving the quality of RA.Among them,the vertical eccentric wheel high speed grinding method and horizontal rotary grinding method in Japan and the particle shaping and strengthening method proposed by Professor Li Qiuyi in China are the obvious methods to improve the effect.

(1) Vertical eccentric high speed grinding method and horizontal rotary grinding method

Japan is relatively advanced in the process of processing RA. It mainly uses vertical eccentric and horizontal rotary devices. The working principle of the vertical eccentric wheel high-speed grinding method is shown in Figure 1. The preliminarily broken concrete block is poured into the bucket, and the eccentric wheel rotates at high speed to squeeze and grind the material to reduce the old cement paste adhering to the surface of the aggregate, and to grind off the more prominent edges and corners on the aggregate, thereby improving the performance of RA[9].

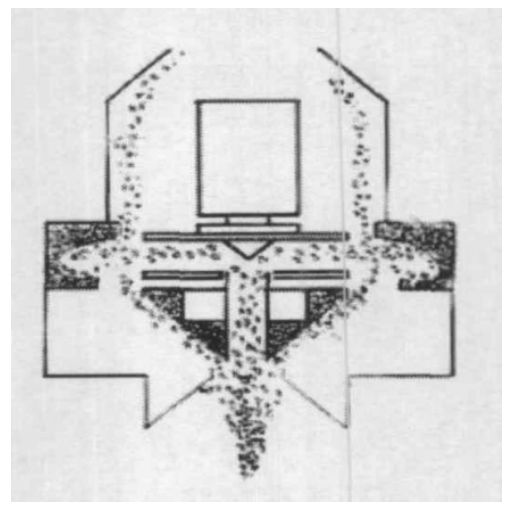

Fig. 1 Vertical eccentric high speed grinding device

The horizontal rotary grinding device is shown in Figure 2. It is similar to the inclined spiral conveyor, except that the spiral blade is transformed into a spiral belt with grinding blocks, and a large number of wear-resistant liners are also arranged on the inner wall of the casing. And at the top of the spiral belt is equipped with a cone that turns opposite to the spiral belt to increase the grinding effect on the material[10].
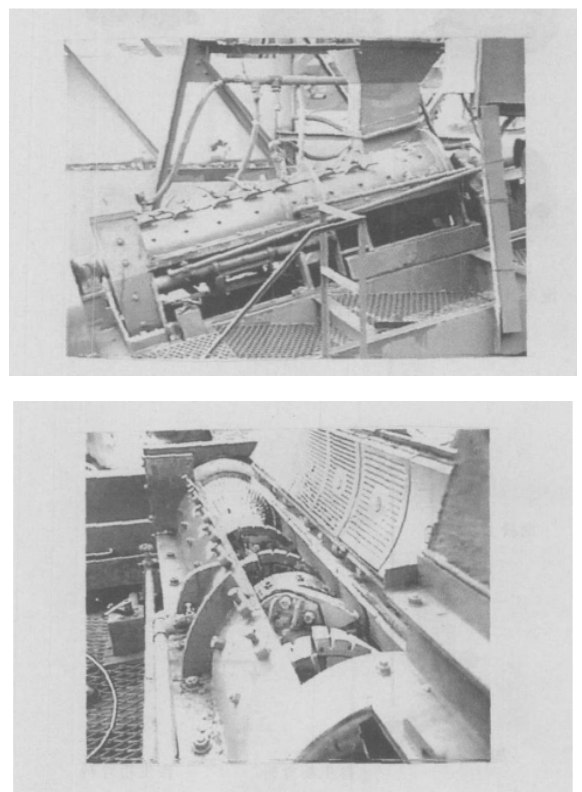

Figure 2 Horizontal rotary grinding equipment

(2)The particle shaping and strengthening method

The particle shaping and strengthening method[11] is proposed by Li Qiuyi. It uses the collision and friction between high-speed moving particles to effectively improve the particle size and remove the old mortar attached to the surface of the RA. The device is shown in Figure 3 . When working, recycled aggregate is added to the machine from the upper port, part of the material enters the interior of the impeller through the top, and is ejected at high speed by the centrifugal action of the impeller when it rotates. The remaining materials fall along the periphery of the impeller and collide with the ejected materials to achieve the purpose of crushing. The crushed material repeatedly hits, cuts and rubs in the cavity many times, so as to achieve the purpose of shaping and strengthening.

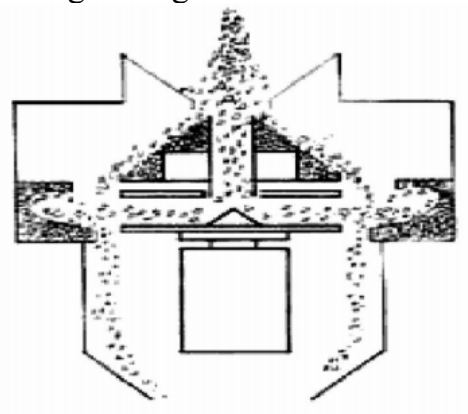

Figure 3 Structure of particle shaping equipment

\subsubsection{Heating grinding method}

Under the action of high temperature, the calcium hydroxide in the cement mortar will be decomposed into 
calcium oxide by heating, and the CSH gel will be dehydrated and deteriorated, so the strength of the mortar will decrease. At the same time, the thermal expansion coefficient of coarse aggregate and mortar is different,the thermal expansion coefficient of cement mortar is larger. When the temperature rises, the volume expansion of cement mortar is larger than that of coarse aggregate. When the temperature rises, the volumetric expansion of cement mortar is larger than that of coarse aggregate, compressive stress is formed in the transition zone between the aggregate and mortar. When the temperature is lowered, tensile stress is formed. The existence of tensile stress and compressive stress will make the interface between aggregate and mortar easier to be destroyed[12]. At high temperatures, the decrease in the strength of cement mortar and the presence of the interface stress between the aggregate and the mortar are all conducive to the separation of the old mortar from the recycled aggregate. Heating tools mainly include microwave ovens and muffle stoves, after heating, the separation of mortar and aggregate is not complete, and mechanical equipment is generally required for grinding.

Sui Yuwu's research[13] found that when heating and grinding recycled aggregates, the heating temperature is lower than $300^{\circ} \mathrm{C}$, which cannot achieve the effect of separating the old mortar and aggregate. While when heating temperature higher than $500^{\circ} \mathrm{C}$, it will cause high temperature damage to the aggregate and significantly increase energy consumption.

Akbarnezhad[14] used high-power microwave (10kw) to heat the RA. The experiment found that thermal stress will be generated between the interface of attached mortar and aggregate during heating,which will delaminate the mortar and aggregate,However, it is found that too high power will cause high temperature damage and cause a certain degree of damage to the original aggregate.

Xiao Jianzhuang[15] used low-power microwaves to cyclically heat the recycled aggregates. The aggregates were heated at $800 \mathrm{~W}$ for 5 minutes, then taken out and placed in cold water for cooling. After cooling, they continued to be placed in a microwave oven and circulated back and forth. Experiments have found that microwave heating cycle is better than mechanical grinding, and the water absorption and crushing value decrease more obviously. The $28 \mathrm{~d}$ strength of the recycled aggregate concrete after microwave strengthening is similar to that of ordinary concrete.

The general process of the heating grinding method is as fpllow: after the waste concrete is initially crushed, the crushed materials are put into a heating device for heating treatment to dehydrate and embrittle the cement stone attached on the aggregate, and then the aggregate and cement stone are separated by grinding of the mechanical device. The heating grinding method can significantly improve the performance of RA. Some Japanese factories use the heating grinding method, heating for 60 minutes and then grinding for 120 minutes, which can significantly reduce the amount of mortar attached on the RA surface, and meanwhile can significantly reduce the porosity and water absorption of the RA.
On the basis of heating grinding, the selective heating grinding method is also proposed. Bru[16] adopted selective heating and grinding method, microwave heating treatment was carried out selectively at the junction of aggregate and old mortar, combined with mechanical grinding, it obtained good quality recycled aggregate.

Under the action of mechanical force, the old cement paste is removed, and finally high-purity crushed stone is obtained. However, the technology consumes more energy and resources, requires complex equipment, which leads to higher cost, and the recycled aggregate is often worn seriously, which is not conducive to the long-term performance of the concrete.Nevertheless, mechanical processing of recycled aggregates is still the main method of pretreatment of RA.

\subsection{Chemical strengthening method}

Chemical strengthening method is to soak RA in a specific chemical solution, through the action of the physical properties of the solution or the reaction between the solution and the cement stone composition, to achieve the purpose of strengthening the RA.The mechanism of solution impregnation can be divided into three categories, one is to weaken the old mortar, make the old mortar deteriorate and easy to separate from aggregate, the second is to strengthen the old mortar, repair crackles and fill cracks; the third is to improve the surface properties of the aggregate, such as forming a film on the surface of the aggregate, which can well reduce the water absorption. According to the difference of chemical solution, it can be divided into inorganic solution impregnation, organic solution impregnation and cement slurry impregnation.

\subsection{1 inorganic solution impregnation}

\section{(1)acid solution impregnation}

The hydration products of the cement in the recycled aggregate can react and dissolve in the acid solution, which is beneficial to the separation of the old mortar and the primary aggregate. Acid solution impregnation is a simple modification method, which can adjust the modification effect of recycled aggregate by adjusting the type, concentration and impregnation time of acid. The commonly used acids are hydrochloric acid, sulfuric acid, phosphoric acid and glacial acetic acid.

Tam V W[17] comprehensively studied the dissolution of the attached mortar after being impregnated with three different acid solutions of hydrochloric acid, sulfuric acid, and phosphoric acid for 24 hours.Experiments have found that various of acids can react with the cement hydration products attached to the RA, which will dissolve the old mortar, so it can reduce the water absorption rate and improve the compressive and flexural strength of the recycled aggregate concrete. Among them, the hydrochloric acid works best.

Hydrochloric acid has a relatively obvious modification effect on RA[18], the concentration of 
impregnation is generally $0.1-0.5 \mathrm{~mol} / \mathrm{L}$, and the impregnation time is less than 3 days. When using acid solution to modify RA, if over-treatment (too high concentration or too long immersion time), it will weaken the surface of the aggregate and increase the number of pores, which may damage the original aggregate. Güneyisi E[19] used SEM to analysis and found that the $0.1 \mathrm{~mol} / \mathrm{L} \mathrm{HCl}$ can better remove the adhered mortar and some loose substances on the surface of the RA, and at the same time, it will not cause obvious damage to the aggregate surface.

Impregnation with acid solution also has disadvantages. It will generate much salt substances such as sulfate ions and chloride ions, which will affect the durability and permeability of recycled aggregate concrete, while glacial acetic acid (acetic acid) is used, the acetate ion generated will not affect the durability of concrete.Compared with other strong acids, acetic acid has obvious advantages, including lower cost, safer operation, and cleaner process.Acetic acid can react with the hydration products in the attached mortar to produce calcium acetate, and calcium acetate can improve the early performance of concrete. Pan $Z[20]$ found that when the acetic acid concentration is $0.2 \mathrm{~mol} / \mathrm{L}$ and the soaking time is $24 \mathrm{~h}$, the performance of the recycled aggregate was improved the most. Using the soaked solution as the mixing water could slightly extend the setting time of the cement slurry and increase the initial fluidity and fluidity loss, from a practical perspective, the impact is within an acceptable range.

(2) Sodium silicate solution (water glass) impregnation

When soaking RA with sodium silicate solution (water glass), on the one hand, sodium silicate can react with $\mathrm{Ca}(\mathrm{OH}) 2$ in the mortar to form hydraulic calcium silicate colloid, on the other hand, silica acid gel will precipitate after the sodium silicate solution hardens. These two substances can fill the capillary pores, repair the micro cracks on the surface, and form a smooth and hard coating on the surface of the aggregate,which can reduce the water absorption and crushing value of the aggregate.

Gesoğlu's[19] research found that prepreg treatment of the water glass has a very obvious effect on reducing the water absorption. It will form a smooth, strong and dense external coating on the surface of the aggregate, and the water absorption of RA will reduce to about $2 \%$.

Yang L X found[21] that the water absorption rate of RA would decrease with the increase of sodium silicate concentration, but the excessive concentration of sodium silicate would thicken the concrete mixture, thus increasing the water consumption of recycled aggregate concrete. At the same time, he studied the influence of the drying method and soaking time of sodium silicate on the water absorption of RA, and found that the water absorption first increases and then decreases as time goes by,soaking for 1 hour has the largest decrease in water absorption, and drying at $25^{\circ} \mathrm{C}$ has a more significant effect on reducing the water absorption of RA than drying at $60^{\circ} \mathrm{C}$.

Pre-treatment of reclaimed aggregate with sodium silicate solution can significantly reduce water absorption, but the introduction of sodium ions will increase the risk of alkali aggregate reaction, which can be inhibited by adding silica powder

\subsubsection{Impregnation with organic solution}

\section{(1)Organic polymer emulsion}

Organic polymer emulsion has good bonding performance and can be cured in a short time. After RA is immersed in the emulsion, because the organic polymer has good cohesive force and reactive group activity, it can fill or inhibit the pore structure and micro-cracks. Some organic polymers (such as polyvinyl alcohol emulsion and silicon-based polymers) are hydrophobic and can form a hydrophobic film on the surface of the aggregate, thereby it can reduce the water absorption of RA.Commonly used organic polymer emulsions are: polyvinyl alcohol (PVA), vinyl acetate (EVA), styrene butadiene rubber (SBR), and silane coupling agent solutions and so on.

Kou[22] used PVA solution to treat RA, and the physical and mechanical properties of RA had been significantly improved. The results showed that as the PVA concentration increases, the water absorption rate of RA decreases, the recommended concentration of PVA impregnation is $10 \%$. PVA emulsion has obvious precipitation effect on recycled aggregates, but due to its large molecules, it is not easy to enter the inside of the aggregates, so it needs to be pressed in with a vacuum pump under the action of external pressure when using[23]. The recycled aggregate can also be treated by mixing polymer emulsion, such as mixing acid solution and EVA emulsion, they do not interfere with each other, which can not only improve the surface of the aggregate, but also reduce the water absorption rate.

In addition, using polymer emulsion to modify the recycled fine aggregate can improve the flexural strength of the mortar, and the flexural and compressive strength ratio is also improved, which is close to the strength level of the mortar prepared by ordinary fine aggregate[24].

\section{(2) Silicone waterproofing agent}

Under the action of water and carbon dioxide in the air, the silicone waterproofing agent self-polymerizes to form a layer of silicone waterproof membrane, which can block part of the pores of the recycled aggregate. In addition, it is hydrophobic and can penetrate into the surface of the recycled aggregate to a certain depth, so ,it increases the contact angle of the surface of the recycled aggregate with water and reduces the water absorption rate of the recycled aggregate[25].

\subsubsection{Cement slurry impregnation}

\section{(1)Pure cement slurry impregnation}

The recycled aggregate is immersed in a plain cement slurry prepared with a higher-strength cement, and then dried.The plain cement slurry penetrates into the aggregate, through adsorption and hydration reaction, it can fill pores, repair cracks and strengthen the old mortar, thereby it can improve the internal structure of 
RA. However, some scholars[15] have found that the performance improvement of recycled aggregate concrete is not very obvious with pure cement slurry immersion through experiments .

(2) Cement mixed slurry impregnation

In addition to pure cement slurry, ultrafine minerals (fly ash, silica fume etc.), waterproofing agent $(\mathrm{FeCl} 3$ waterproofing agent, Kim powder waterproofing agent etc.) or calcium sulfoaluminate expansion agent can also be added to the cement,which Formulated into different cement mixed slurry to soak RA. The particle size of the added material is generally one or two orders of magnitude smaller than that of the cement particles, which can fill the space between the cement particles and improve the compactness of the recycled concrete.Du Ting[26] used four different slurries, plain cement slurry, cement slurry mixed with silica fume , cement slurry mixed with Kim powder, and cement slurry mixed with fly ash, to soak RA. It was found that these kinds of grout can improve the apparent density and aggregate strength of RA to a certain extent, and the treatment of cement mixed with Kim powder has the best effect on strengthening the compressive strength of recycled concrete.

Pozzolanic materials such as fly ash and silica fume have active ingredients, which can cause secondary hydration reactions, to further fill and repair the interior of recycled aggregates, and improve the durability of recycled concrete. Tam's[28] research found that silica fume treatment can improve the micro-structure of the transition zone between the old-new mortar and the aggregate-old mortar in recycled concrete.In general, the efficiency of pozzolanic slurry treatment depends on the particle size, the calcium hydroxide content in the adhesive mortar, the alkalinity of the pore solution and the activity of the pozzolanic material[29].

Impregnating RA with cement slurry will form a layer of slurry on the surface of the aggregate, increasing the thickness of the mortar. While increasing the apparent density and improving the internal structure, it will increase the water absorption rate of RA. However, the mechanical properties of the material are not improved much. In addition, when using recycled aggregates to configure concrete, pozzolanic materials can also be mixed in the form of mineral admixtures

\subsection{Carbonization treatment}

Carbonization treatment refers to the strengthening of RCA with $\mathrm{CO}_{2}$. It is based on the carbonization of concrete and $\mathrm{CO}_{2}$ mineralization and storage technology. $\mathrm{CO}_{2}$ can react with $\mathrm{Ca}(\mathrm{OH})_{2}$ and $\mathrm{C}-\mathrm{S}-\mathrm{H}$ in the mortar attached to the recycled aggregate, and the reaction products are mainly calcium carbonate and silica gel[30]. The main reaction equation is as follows:

$$
\mathrm{Ca}(\mathrm{OH}) 2(\mathrm{~s})+\mathrm{CO} 2 \rightarrow \mathrm{CaCO} 3(\mathrm{~s})+\mathrm{H} 2 \mathrm{O}(\mathrm{l})
$$

$$
\mathrm{C}-\mathrm{S}-\mathrm{H}(\mathrm{s})+\mathrm{CO} 2(\mathrm{~g}) \rightarrow \mathrm{CaCO} 2(\mathrm{~s})+\mathrm{SiO} 2 \cdot \mathrm{nH} 2 \mathrm{O}(\mathrm{s})
$$

The reaction products fill between the pores, increasing the apparent density of RA, reducing the porosity, improving the compactness of the attached slurry, making the RA structure denser and reducing its water absorption. In addition, $\mathrm{CO}_{2}$ can also react with the mineral components $\left(\mathrm{C}_{2} \mathrm{~S}, \mathrm{C}_{3} \mathrm{~S}\right)$ in the cement particles that are not involved in the hydration reaction in the attached mortar, which increase the volume of the solid phase[31].

Under the condition that the $\mathrm{CO}_{2}$ concentration exceeds $99 \%$ and the pressure is $0.1 \mathrm{bar}$, kou had studied the influence of different carbonization curing time (6, $12,24,48,72 \mathrm{~h}$ ) on RA, and found that the longer the carbonization curing time, the lower the water absorption of RA, the better the aggregate quality.But when $72 \mathrm{~h}$ compared with $48 \mathrm{~h}$, the properties of RA changed little[32].In the process of curing concrete by $\mathrm{CO}_{2}$, there is a maximum value for the improvement of concrete performance[33].

$\mathrm{CO}_{2}$ reinforced recycled concrete aggregate is affected by humidity, $\mathrm{CO}_{2}$ concentration, pressure, time and temperature as well as the properties of recycled aggregate (water content, particle size, aggregate strength). After carbonization of aggregate, the mechanical properties and durability of recycled aggregate concrete can be significantly improved.

When $\mathrm{CO}_{2}$ strengthens recycled concrete aggregates, it is affected by humidity, $\mathrm{CO}_{2}$ concentration, pressure, time and temperature, and the properties of recycled aggregates (water content, particle size, aggregate strength).After carbonizing of RA, it can obviously improve the mechanical properties and durability of recycled aggregate concrete[34].

Carbonization treatment is a new type of aggregate strengthening treatment technology, which can significantly improve the surface properties of RA and improve the performance of recycled concrete.However, the technology has not yet been put into actual production, further research is needed on the internal structure of the concrete and the corrosion of the steel bar with carbonization treatment. .

\subsection{Nano material modification treatment}

Nano-materials refers to the ultra-fine materials with a size of $1-100 \mathrm{~nm}$, it has the characteristics of small size, strong permeability, large specific surface area, and many active atoms. Take active nano $\mathrm{SiO}_{2}$ as an example,when mixed into concrete, it can fill the pore of aggregate, compact structure; and it is active and can react with calcium hydroxide to form $\mathrm{C}-\mathrm{S}-\mathrm{H}$ gel; while it has a crystal nucleation effect, which connects the sparsely distributed hydration products into a denser network structure. Thereby, it can optimize ITZ and improve the strength and durability of concrete[35]. When the recycled aggregate is soaked in the slurry which mixed with nano material, it can fill the voids, optimize the surface of the aggregate, finally improve the performance of the aggregate.

Some scholars use nano- $\mathrm{SiO}_{2}$ solution to soak the recycled aggregates. Experiments have found that the durability of the modified recycled aggregate concrete is improved, and the mechanical properties are close to 
natural concrete. The flexural and shear strength of the beam members which made of the recycled aggregate modified by nanomaterials are improved to a certain extent[36,37].

Nanomaterials can also be mixed into recycled concrete in the form of admixtures. Yang Qing[38] mixed high-activity nano- $\mathrm{SiO}_{2}$ and nano-modified mineral admixtures into recycled concrete. The results show that the combination of the two materials can better fill the microscopic pores in the transition zone between the new and the old interface, increase the strength of the ITZ, and enhance the internal compactness of the concrete.For that, the strength and impermeability of recycled concrete are improved

Nanomaterials can reduce the amount of cement, improve the strength and durability of recycled concrete, especially the early performance, these are all based on the optimization of microstructure and the promotion of hydration reaction by nanomaterials. While, nano-materials are easy to agglomerate and are difficult to stir evenly, which has a great impact on the workability of the fresh concrete. Moreover, nanomaterials are generally more expensive. so the use of nanomaterials to pretreat recycled aggregates cannot be promoted and applied, and the content of nanomaterials is generally less than $7 \%$ of the total amount of cementitious materials[39,40].

\subsection{Microbial mineralization and bio-deposition technology}

Microorganisms can produce calcite through mineralization and deposition during metabolism (carbonate mineral, $\mathrm{CaCO} 3$ ) during metabolism. Boquet[41] discovered this phenomenon for the first time in 1973, in his experiment, it was found that soil bacteria could induce the deposition of calcium carbonate minerals. In 1999, Stocks[42] applied the phenomenon of Microbial mineralized deposits to the repair of concrete cracks. Microbial mineralization and bio-deposition technology is a new technology for treating RA. It uses the minerals produced by microbial bio-mineralization to fill or bond the pores of RA, so it can improve the pore structure and repair the micro-cracks on the surface to strengthen the material[43,44].Generally, after the appropriate strains are grown with the culture medium, they are formulated into a bacterial solution to soak the RA with a treatment seal.

The basic advantage of it is that the bacteria is small in size and can enter the micro-pores that cement or fly ash cannot enter. Grabiec's[45] research found that microbial treatment technology can improve the surface structure of RA and reduce the water absorption of recycled concrete aggregates. Zhu[46,47] used DSM8715 strain culture solution to soak the recycled coarse and fine aggregates. Through XRD and SEM experiments, it was found that the strains would form calcite-type calcium carbonate precipitates on the aggregates, which reduced the water absorption rate and crushing index of the aggregates. $\mathrm{Wu}[48]$ treated $\mathrm{RA}$ with pseudobacillus (DSM8715) and found that the smaller the RA's particle size, the more the water absorption of the aggregate decreases, and the less the aggregate crush value decreases. It may be related to the proportional formation of calcium carbonate on the surface of the aggregate

While microbial mineralization and bio-deposition technology improves the quality of recycled aggregates, it does not pollute the environment and consumes less energy. At present, this microbial deposition process has been applied to a certain extent in the repair and durability improvement of building materials[49]. However, the process of microbial mineralization and deposition to generate calcium carbonate is a slow growth and metabolism process, when it is used to modify the recycled aggregate, it is difficult to penetrate into the aggregate which takes a long time period, generally about 20 days. So in order to popularize the application, we need to study the method of shortening the time

\subsection{Water cleaning treatment}

The water cleaning treatment method is mainly to wash the RA with water, which can wash away the impurities, loose particles and old mortar with weak adhesion in the aggregate. It is generally treated by rinsing or soaking in circulating water.

A method of water cleaning is mentioned in the literature[50]. The recycled aggregate is placed in the rotating mill drum, the volume of which is about one third of the volume of the rolling mill drum. The drum rotates at about $60 \mathrm{r} / \mathrm{min}$ for 10 to 15 minutes, and then soaks aggregate with water. Compared with the general water cleaning method, this method can improve the performance of aggregate better. Katz[51] used the repeated ultrasonic washing method to wash the recycled aggregate in a water bath, and found this method can remove the weakly adhered mortar, the compressive strength of the recycled aggregate concrete after the ultrasonic modification treatment increased by $7 \%$. Meanwhile, it was found that compared with high-grade concrete, low-grade concrete would produce more fine particles when it is broken, which requires more cleaning cycles to clean the recycled aggregates.ELhakam[52] tried to soak RA in water for up to 30 days to improve the quality of RCA. The results showed that after immersion treatment, the $7 \mathrm{~d}$ and $56 \mathrm{~d}$ compressive strength of concrete increased by $4.8 \%$ and $15.7 \%$ respectively.

Compared with other modified and strengthened methods, water cleaning is easier to achieve, and the operation is simple, but it can only wash away loose loose particles and mortar with weak adhesion, and the optimization performance is limited. At the same time, if there is no thorough recycling process, it will consume much water resources and cause certain pollution to the environment. Water cleaning treatment methods can generally be used in combination with other treatment methods. 


\section{3 conclusion}

At present, there are many modification methods of RA, among which physical method is the most commonly used, but it has high energy consumption and great wear on the recycled aggregate. The chemical method has a simple process, but the influence of some possible reactions on the durability is not clear, so it has not been applied in engineering practice on a large scale. I believes that the incorporation of pozzolan materials can effectively fill the pores of the mortar on the recycled aggregate and the voids in the interface transition zone, causing secondary hydration reactions, improving the interface transition zone of recycled aggregate concrete, and the cost is low. It is very suitable for improving the performance of recycled aggregate and recycled concrete. I believe that adding pozzolanic material can effectively fill the pores of the adhesive mortar on the recycled aggregate and the gap of the ITZ, which can lead to the secondary hydration reaction, it can improve the ITZ of the recycled aggregate concrete, and the cost is low, so it is a very suitable method for improving the performance of the recycled aggregate and recycled concrete.

Nano-material modification is relatively expensive, and it is impossible to get large-scale application. Carbonization treatment is a very potential method, which can not only improve the quality of recycled aggregate to improve the mechanical properties and durability of recycled concrete, but also reduce carbon emissions. It has high economic and environmental benefits. However, there are still problems such as the collection of industrial $\mathrm{CO} 2$, and it is still in the experimental research stage. The process of microbial modification has no pollution and low energy consumption, although it takes a long time, it is still a method worthy of further study.

At present, most of the experiments and studies on the modification of recycled aggregate focus on the study of mechanical properties and various short-term indicators. We should consider the long-term use performance of recycled concrete and conduct further research on its strength stability and durability.

\section{References}

1. Su Wenyang, Hong Li, Li he Shan . Engineering and Construction . 2020, 34(06): 1197-1200.

2. Yao Yao, Liu Hongmei, Wang Jianjun, Mile, Li Huanhuan..Journal of Materials Science and Engineering. 2019(02)Page:339-344

3. Ye Ting. The Performance of Modified Pervious Asphalt Concrete with Recycled Coarse Aggregate.[D] Nanchang University ,2020.

4. Gutiérrez A. International RILEM Conference on the Use of Recycled Materials in Building and Structures. RILEM Publications SARL, 2004: 536-544.

5. Xiao Jianzhuang, Liu Qiong, Li Wengui. Journal of Qingdao Technological University. 2009(04) Page:24-30
6. Yi Chao. The Study on the Resource Recovery of Construction Waste for Recycled Aggregate Concrete[D].Jinan University,2014.

7. Peng GaiFei, Huang Yanzhu, Zhang Jiufeng. Journal of Building Materials. 2012(01) Page:80-84

8. Li Qiuyi Concrete recycled aggregate [M]. China Architecture \& Building Press.2011.2

9. Zhan Xiangyu, Gao Ruize. Construction Wall Innovation \& Building Energy-Saving. 2019(12) Page:67-69

10. Li Qiuyi, Li Yunxia, Zhu Chongji, Tian Li.Concrete. 2006(01) Page:74-77

11. Li Qiuyi, Li Yunxia, Zhu Chongji. Materials Science and Technology. 2005(06) Page:579-581+585

12. Wu Xubiao, Zhou Guangli. Journal of Guizhou University of Finance and Economics. 2020(04) Page: $147-150$

13. Sui Yuwu, Tian Qingbo, Yue Xuetao, Zhang Fengqing. Journal of Shandong Jianzhu University. 2015(06) Page:544-549

14. A. Akbarnezhad, K.C.G.Ong, M.H.Zhang, et al. Construction and Building Materials, 2011, 24(8):3469-3479.

15. Xiao Jianzhuang, Wu Lei, Fan Yuhui. Concrete. 2012(07) Page:55-57

16. Bru K, Touzé S, Bourgeois F, et al. International Journal of Mineral Processing, 2014, 126: 90-98.

17. Tam V W Y, Tam C M, Le K N. Resources, Conservation and Recycling, 2007, 50(1): 82-101.

18. Kim Y, Hanif A, Kazmi S M S, et al. Journal of cleaner production, 2018, 191: 339-349.

19. Güneyisi E, Gesoğlu M, Algın Z, et al. Construction and Building Materials, 2014, 64: 172-183.

20. Pan Z, Wang S, Liu Y, et al. Journal of Cleaner Production, 2019, 235: 866-874.

21. Yang L X, Yao-Li Q, Fang Q Q, et al. 3rd International Conference on Material Engineering and Application (ICMEA 2016). Atlantis Press, 2016: 181-186.

22. Kou S C, Poon C S. Cement and Concrete Composites, 2010, 32(8): 649-654.

23. Wei Yipeng, Ye Qiaoling, Guo Zirui. China Southern Agricultural Machinery. 2019(04) Page: $198+231$

24. Wang Ziming, Pei Xuedong, Wang Zhiyuan. CONCRETE. 1999(02) Page:44-47

25. Chen Deyu, Yuan Wei, Liu Huan. New Building Materials. 2009(02) Page:20-23

26. Du Ting, Li Huiqiang, Wu Xianguo.New Building Materials,2002(03):6-8.

27. S.C. Kou; C.S. Poon (2012).Enhancing the durability properties of concrete prepared with coarse recycled aggregate. , 35(none), 69-76.

28. Vivian W.Y. Tam X.F. Gao; C.M. Tam (2005). Microstructural analysis of recycled aggregate concrete produced from two-stage mixing 
approach. , 35(6), 1195-1203.

29. Singh, L.P.; Karade, S.R.;Bhattacharyya, S.K.; Yousuf, M.M.; Ahalawat, S. (2013).Beneficial role of nanosilica in cement based materials - A review. Construction and Building Materials, 47, 10691077.

30. Li Linkun, Liu Qi, Ma Zhong, Peng Bo. Thermal Power Generation. 2021(01)Page:94-103

31. Shi C, Li Y, Zhang J, et al. Journal of Cleaner Production, 2016, 112: 466-472.

32. Kou S C, Zhan B, Poon C S. Cement and Concrete Composites, 2014, 45: 22-28.

33. Zou Qingyan. Carbon dioxide curing concrete technology research [D].Central South University,2008.

34. Pu Yunhui, Shi Xiaoshuang, Wang Qingyuan, Li Wenyuan, Fu Ling.Concrete,2020(06):65-69.

35. Yan Hongsheng. Experimental Study on Mechanical Properties of Recycled Coarse Aggregate Concrete Strengthened with Nano SiO2[D].Qingdao Technological University,2018.

36. Zhang $\mathrm{H}$, Zhao $\mathrm{Y}$, Meng $\mathrm{T}$, et al. Journal of Materials in Civil Engineering, 2016, 28(2): 04015094.

37. Zhu Yongnian, Zhang Hongru, Meng Tao, Zhao Yuxi. Concrete 2014(07):138-144.

38. Yang Qing, Qian Xiaoqian, Qian Kuangliang, Wang Zhangfu, Zhou Tanggui. Journal of Materials Science and Engineering. 2011,29(01):66-69+130.

39. Wang Yujie, Liu Binghua. Shandong Communications Technology. 2014(02):39-41.

40. Meng Tao, Qian Kuangliang, Qian Xiaoqian, Zhan Shulin. Rare Metal Materials and Engineering. 2008, 37(S2):667-669.

41. Boquet E, Boronat A, Ramos-Cormenzana. Nature, 1973, 246(5434): 527-529.

42. Stocks-Fischer S, Galinat J K, Bang S S. Soil Biology and Biochemistry, 1999, 31(11): 1563-1571.

43. Zhang Xiaotong. [D].Qingdao Technological University,2016.

44. Wu Chunran. [D].Qingdao Technological University,2018.

45. Grabiec A M, Klama J, Zawal D, et al. Construction and Building Materials, 2012, 34: 145-150.

46. Zhu Yaguang, Wu Yankai, Wu Chunran. China Concrete and Cement Products,2017(12):93-96.

47. Zhu Yaguang, Wu Chunran, Wu Yankai, Xu Peizhi. Concrete,2018(07):88-92.

48. Wu C R, Zhu Y G, Zhang X T, et al. Cement and Concrete Composites, 2018, 94: 248-254.

49. N.K. Dhami, A. Mukherjee, M.S. Reddy, Biofilm and Microbial applications in Biomineralized concrete, in: Jong Seto (Ed.), Adv. Top. Biominer, InTech, 2012,pp. 137-164.

50. Mistri Abhijit; Bhattacharyya, Sriman Kumar;
Dhami, Navdeep; Mukherjee, Abhijit; Barai, Sudhirkumar V. (2020). Construction and Building Materials, 233(), 117894

51. Katz, Amnon (2004).Treatments for the Improvement of Recycled Aggregate. Journal of Materials in Civil Engineering, 16(6), 597-603.

52. Ali Abd Elhakam; Abd Elmoaty Mohamed; Eslam Awad (2012).Influence of self-healing, mixing method and adding silica fume on mechanical properties of recycled aggregates concrete.,35(none) 